\title{
A rat model of post-traumatic stress disorder reproduces the hippocampal deficits seen in the human syndrome
}

\author{
Sonal Goswami ${ }^{1}$, Sherin Samuel ${ }^{1}$, Olga R. Sierra ${ }^{1}$, Michele Cascardi ${ }^{1,2}$ and Denis Paré ${ }^{1 *}$ \\ Center for Molecular and Behavioral Neuroscience, Rutgers State University, Newark, NJ, USA \\ ${ }^{2}$ Montclair State University, Montclair, NJ, USA
}

\author{
Edited by: \\ Sumantra S. "Chattarii," National \\ Centre for Biological Sciences, India \\ Reviewed by: \\ Christa Mclntyre, University of \\ Texas, USA \\ Gregory Quirk, University of Puerto \\ Rico, USA

\section{*Correspondence:} \\ Denis Paré, Center for Molecular \\ and Behavioral Neuroscience, \\ Aidekman Research Center Rutgers, \\ The State University of New Jersey, \\ 197 University Avenue, Newark, \\ NJ 07102, USA. \\ e-mail: pare@andromeda. \\ rutgers.edu
}

\begin{abstract}
Despite recent progress, the causes and pathophysiology of post-traumatic stress disorder (PTSD) remain poorly understood, partly because of ethical limitations inherent to human studies. One approach to circumvent this obstacle is to study PTSD in a valid animal model of the human syndrome. In one such model, extreme and long-lasting behavioral manifestations of anxiety develop in a subset of Lewis rats after exposure to an intense predatory threat that mimics the type of life-and-death situation known to precipitate PTSD in humans. This study aimed to assess whether the hippocampus-associated deficits observed in the human syndrome are reproduced in this rodent model. Prior to predatory threat, different groups of rats were each tested on one of three object recognition memory tasks that varied in the types of contextual clues (i.e., that require the hippocampus or not) the rats could use to identify novel items. After task completion, the rats were subjected to predatory threat and, one week later, tested on the elevated plus maze (EPM). Based on their exploratory behavior in the plus maze, rats were then classified as resilient or PTSD-like and their performance on the pre-threat object recognition tasks compared. The performance of PTSD-like rats was inferior to that of resilient rats but only when subjects relied on an allocentric frame of reference to identify novel items, a process thought to be critically dependent on the hippocampus. Therefore, these results suggest that even prior to trauma PTSD-like rats show a deficit in hippocampal-dependent functions, as reported in twin studies of human PTSD.
\end{abstract}

\footnotetext{
Keywords: post-traumatic stress disorder, animal model, recognition memory, extinction, predatory threat, elevated plus maze, open field
}

\section{INTRODUCTION}

Exposure to a severely traumatic event leads to the expression of a syndrome termed post-traumatic stress disorder (PTSD), which is observed in $28-50 \%$ of known trauma survivors (e.g., combat experience, crime victims) and affects around $7 \%$ of the population (Nemeroff et al., 2006). The development and expression of PTSD is a complex process, involving predisposing factors along with physiological, behavioral, emotional, and cognitive changes that emerge after trauma (Nugent et al., 2008; Afifi et al., 2010). However, progress in identifying these factors has been hampered by ethical limitations associated with human research. For example, humans cannot be randomly assigned to trauma, and importantly, the invasive techniques required to study the pathophysiology of PTSD can only be used in animals. Thus, a promising approach toward understanding the pathophysiology of PTSD would be to study the disease in a valid animal model of the human syndrome.

To this end, we have begun testing the validity of a rat model of PTSD. In this model, Lewis rats are exposed to a predatory threat. Importantly, this threat does not cause a general increase in anxiety expressed by all rats (Goswami et al., 2010). Instead, it leads to extreme and persistent behavioral manifestations of anxiety in a subset of rats (termed PTSD-like rats), while other rats (called Resilient) show no long-term consequences of the stressor (Cohen et al., 2006a,b). Compared to Resilient rats, PTSD-like rats are impaired at the extinction of classically conditioned fear responses (Goswami et al., 2010). Importantly, as in human PTSD (Milad et al., 2008), this impairment develops as a result of trauma exposure and does not predate it (Goswami et al., 2010). While these results are promising, further testing of the model's validity is warranted.

Human imaging studies indicate that individuals with PTSD have smaller hippocampal volumes (Gilbertson et al., 2002; Bremner et al., 2003; Kitayama et al., 2005; Wang et al., 2010) and exhibit impaired performance on hippocampal-dependent tasks (for instance, see Shin et al., 2004; Lindauer et al., 2006; Gilbertson et al., 2007; Thomaes et al., 2009; Hayes et al., 2011; reviewed in Samuelson, 2011). Interestingly, studies of monozygotic twins discordant for combat exposure have revealed that these hippocampal abnormalities were present in identical twins that had not been exposed to trauma, suggesting that they predate onset of the disorder (Gilbertson et al., 2002, 2007). Therefore, the present study was undertaken to test whether the Lewis rat model of PTSD reproduces the hippocampal-dependent behavioral deficit observed in human PTSD, and whether it can be detected before the rats are exposed to predatory threat. 
Thus, prior to predatory threat, we tested separate groups of Lewis rats on three types of recognition memory tasks that varied in the types of clues the rats could use to identify item novelty: identity of the objects or their location in ego- or allo-centric coordinates. Since the latter is known to depend on hippocampal functioning (Langston and Wood, 2009), one would expect rats predisposed to PTSD to show greater recognition deficits when identifying item novelty based on allocentric encoding. After the completion of the tasks, rats were exposed to predatory threat, and long-term anxiety-like behavior was assessed 7-d later on the elevated plus maze (EPM). As in previous studies (Cohen et al., 2006a,b; Goswami et al., 2010), exploratory behavior on the EPM was used to classify rats as PTSD-like (rats that spent zero time in the open arms) vs. Resilient (rats that explored the open arms).

\section{MATERIALS AND METHODS}

All procedures were approved by the Institutional Animal Care and Use Committee of Rutgers University, in compliance with the Guide for the Care and Use of Laboratory Animals (DHHS). Our subjects were male Lewis rats (starting weight of 225-250 g; Charles River Laboratories, New Field, NJ) that were housed individually. The rats had continuous access to food and water and were maintained on a $12 \mathrm{~h}$ light/dark cycle. Prior to the experiments, they were habituated to the animal facility for one week.

\section{OPEN FIELD TEST}

Performance on the object memory tasks used here not only depends on visual, mnemonic, and spatial orientation abilities, but also anxiety levels and exploratory behavior. Thus, to assess whether Resilient vs. PTSD-like rats differ in these two latter respects, we tested a group of naïve Lewis rats $(n=81)$ in the open field $(\mathrm{OF})$ prior to predatory threat. Our OF apparatus was a square box $(1 \times 1 \mathrm{~m})$ surrounded by walls, $60 \mathrm{~cm}$ high. Red lines drawn on the floor divided the environment in 36 squares of equal area. Rats were placed one at a time at the center of this novel environment and left to explore it under dim light for $5 \mathrm{~min}$. Their exploratory behaviors were recorded on videotape. The next day, the rats were subjected to predatory threat, and one week later, tested on the EPM, as described below.

\section{OBJECT AND OBJECT-PLACE RECOGNITION TASKS}

We subjected different samples of Lewis rats to one of three tasks commonly used to assess various forms of recognition memory in rodents (e.g., Mumby, 2001; Langston and Wood, 2009). In these tasks, preferential exploration of the novel relative to the familiar items is used to assess recognition memory. In each of the three tasks, rats were subjected to a 5 min sample phase and a 5 min test phase, separated by a 2 min inter-phase interval during which they were returned to their home cage. In the first task, the novel object recognition task (NOR), rats were exposed to (and could freely explore) two identical novel objects. Subsequently, during the inter-phase interval, one of the previously explored objects (i.e., familiar) was replaced with a novel one, and the rats were given $5 \mathrm{~min}$ to freely explore these objects during the test phase. Thus, the rats' exploratory behavior during the test phase was driven by object identity. In contrast, in the next two tasks, the egocentric (EOR) or allocentric (AOR) object recognition tasks, rats were exposed to two distinct and novel objects during the sample phase. During the inter-phase interval, one of the two objects presented during the sample phase was replaced with an identical copy of the remaining object. Thus, the novel location of a previously encountered object should determine the rats' exploratory behavior. However, because the rats' starting positions in the sample and test phases were identical in the EOR task, but different in the AOR task, the rats could rely on different frames of reference to identify the novel location of the familiar object: egocentric in the EOR task and allocentric in the AOR task. Since the latter is dependent on hippocampal functioning (Langston and Wood, 2009), one would expect rats with hippocampal deficits to display less preferential exploration of the novel object-place configuration in the AOR task.

\section{Aspects common to all tasks}

The apparatus and habituation procedures used in the three tasks were identical. All object recognition experiments were conducted in an arena made of black Plexiglas $(76$ by $76 \mathrm{~cm}$, with walls $60 \mathrm{~cm}$ high), under red light illumination. Objects were secured to the floor of the arena with Velcro, $5 \mathrm{~cm}$ away from the corners. Objects used in these tasks included plastic beach toys, mugs, aluminum cans, bottles, and pencil holders. Pilot tests showed that the objects used were of similar interest to Lewis rats. This was confirmed in the actual experiments where the time exploring the different objects during the sampling phase did not differ. In particular, less than $\pm 4 \mathrm{~s}$ deviation was seen between maximal and minimal exploration times of individual objects relative to the average exploration time of all objects used within each task. Active exploration of these objects included behaviors such as touching the objects with the nose or paws. In addition, three-dimensional objects were secured to the walls of the arena to provide the rats with visual spatial cues. On Day 0 , the rats were habituated to the test apparatus for $20 \mathrm{~min}$ with no objects present. This was followed by a second $20 \mathrm{~min}$ period of habituation with two identical objects secured at the northeast and northwest corners. After each trial, the testing arena was wiped clean with a $20 \%$ ethanol solution to eliminate odor cues.

\section{Novel Object Recognition (NOR) task (Figure 1A)}

In this task, the sample and test phases were carried out $24 \mathrm{~h}$ after habituation. Sample phase: two identical objects were secured near the north wall. Rats were placed in the arena facing the south wall (away from the objects) and given $5 \mathrm{~min}$ to explore the objects. Inter-phase interval: rats were placed in their home cage for 2 min. During this time, one object was replaced with a novel object. The location of the replaced object was counterbalanced across subjects. Test Phase: rats were placed back into the arena facing the south wall and given 5 min to explore the objects. A total of 31 rats were subjected to the NOR task.

\section{Egocentric Object-place Recognition (EOR) Task (Figure 1B)}

Starting $24 \mathrm{~h}$ after habituation, each rat experienced one trial per day on four consecutive days. The sample and test phases were separated by a $2 \mathrm{~min}$ interval on each trial. Sample Phase: rats 
A NOR Task

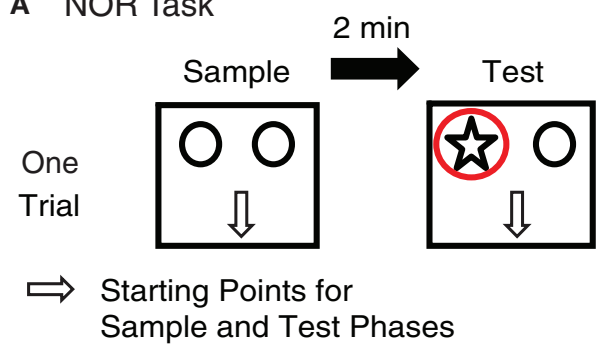

B EOR and AOR Tasks

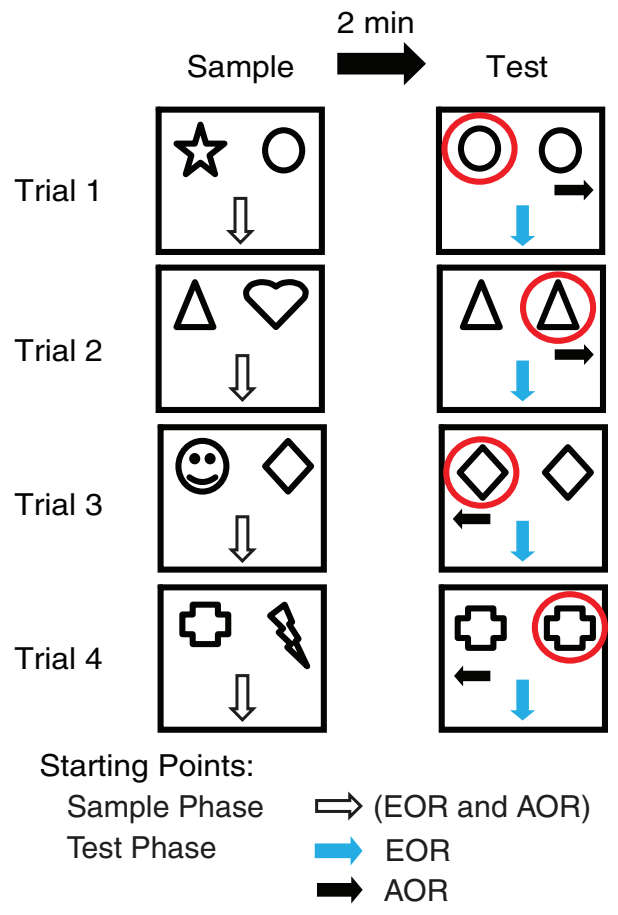

FIGURE 1 | Experimental paradigm. (A) In the novel object recognition (NOR) task, rats were first presented with two identical objects (sample phase, $5 \mathrm{~min}$, left), and returned to their home cage for $2 \mathrm{~min}$. During this period, one of the two objects was replaced with a novel object whose location (east or west) was varied randomly across subjects. During the test phase (right), the rats were allowed to explore the two objects for $5 \mathrm{~min}$. The rats' starting positions in the sample and test phases were identical: facing the south wall. (B) In the egocentric (EOR) and allocentric (AOR) object-place recognition tasks, all aspects of the sample phase were identical: the rats were presented with two novel objects. As for the NOR task, they faced the south wall at the start of this sample phase. After a 5 min exploration period, the rats were returned to their home cage for $2 \mathrm{~min}$. During this period, one object was replaced with an identical copy of the other. The position of this object duplicate was counterbalanced across trials. In the EOR task, the starting position of the rat was identical to that used in the sample phase (facing the south wall, blue arrow). In the AOR task, the rats' starting position varied across trials (facing the east or west wall, black arrow). Four such trials were conducted, on four consecutive days.

were placed in the arena facing the south wall and given 5 min to explore two different objects. Inter-phase Interval: rats were placed in their home cage for $2 \mathrm{~min}$. During this time, one object was replaced by a second copy (duplicate) of the remaining object.
Test Phase: The rats were placed facing the south wall and given $5 \mathrm{~min}$ to explore the new object-place configuration. A total of 36 rats were subjected to the EOR task.

\section{Allocentric Object-place Recognition (AOR) Task (Figure 1B)}

A total of 48 rats were subjected to the AOR task. In this task, all aspects of the protocol were identical to the EOR task except for the positioning of the rats at the start of the test phase. Instead of starting the trials facing the south wall, rats were placed facing either the east or west wall (two trials each) and given 5 min to explore the new object-place configuration. As a result, the only difference between the EOR and AOR tasks is that in the first case the rats could use an egocentric frame of reference to identify the novel item location whereas in the second, they had to rely on an allocentric frame of reference.

\section{Inclusion criteria and measured variables}

In order for the rats' behavior to be considered on a given test trial, they had to have explored both objects for at least $5 \mathrm{~s}$ during the sample phase. In the NOR task, if a subject did not meet this criterion, a second trial was conducted the next day. In the EOR and AOR tasks, if a subject did not meet this criterion, the rat's behavior on that trial was ignored. This occurred rarely (EOR, 2 trials; AOR, 3 trials). Time spent exploring the two objects in the sample and test phases was scored off-line by experienced observers blind to the rat's phenotype, that is, whether the rats had been classified as Resilient or PTSD-like based on their exploratory behavior in the EPM (see below).

Note that the number of trials in the NOR vs. AOR and EOR tasks differs. In the AOR task, because the rats' starting position differs in the sample and test phase, it is standard practice to run four trials, allowing counterbalancing of the difference in starting positions between the sample and test phases as well as left or right location of replaced objects. The same is done in the egocentric version of the object-place task to facilitate comparisons of the results in the EOR and AOR versions of the task. In contrast, the NOR task did not require such a design, as rats always started in the same position.

\section{PREDATORY THREAT}

One day after completion of the tasks, rats underwent predatory threat. Because we wanted to replicate the approach used in two earlier studies on Lewis rats (Cohen et al., 2006a; Goswami et al., 2010), predatory threat involved placing the rats on soiled cat litter ( $48 \mathrm{~h}$ use period; sifted for stool; obtained from male cats) for $10 \mathrm{~min}$ in a standard plastic rat cage with a plastic mesh top. Previously, it was shown that rodents exposed to predators or their odor develops extreme and long lasting ( $\geq 3$ weeks) manifestations of anxiety (Adamec and Shallow, 1993; Blanchard et al., 2003; Adamec et al., 2006). The inherent strength of this species relevant stimulus was demonstrated in studies where predator odor served as an unconditioned stimulus to support cued or contextual fear conditioning (Blanchard et al., 2001; McGregor et al., 2002).

\section{ELEVATED PLUS MAZE (EPM)}

The EPM test was conducted one week after predatory threat. The EPM consisted of four Plexiglas arms $(0.6 \mathrm{~m}$ in length, 
$0.1 \mathrm{~m}$ in width) arranged in a plus pattern, and elevated $0.6 \mathrm{~m}$ from the floor. Two of the arms had a black floor and black walls ( $0.3 \mathrm{~m}$ high). The other two had a white floor and no walls. The animals were placed in the center of the EPM, facing an open arm, and allowed to explore the maze for $5 \mathrm{~min}$ under red light illumination. A video camera positioned above the EPM recorded the rats' behavior.

\section{RESULTS}

\section{BEHAVIOR OF RESILIENT AND PTSD-LIKE RATS IN THE OPEN FIELD}

A total of 81 Lewis rats were tested in the OF, subjected to predatory threat the next day, and tested on the EPM one week later. In this and following experiments, exploratory behavior in the EPM was used to classify the rats in two groups, as initially proposed by Cohen et al. (2006a). Rats that displayed extremely compromised exploratory behavior in the EPM (zero time in the open arms) were classified as "PTSD-like" (44 rats or 54\%) whereas rats that explored the open arms for any amount of time were classified as "Resilient" (37 rats or 46\%). The incidence of the PTSD-like phenotype in this sample is consistent with that found in previous studies using the same paradigm (45-50\%; Cohen et al., 2006a; Goswami et al., 2010), and much higher than in naïve Lewis rats (not subjected to predatory threat; 13\%; Goswami et al., 2010). Importantly, by comparing various measures of anxiety in naïve vs. Resilient rats, the latter study determined that predatory threat did not cause a general increase in anxiety expressed by all subjects, but the emergence of extreme behavioral manifestations of anxiety in a subset of susceptible Lewis rats.

As shown in Table 1, we compared the behavior of PTSD-like and Resilient rats in the OF (prior to predatory threat) along eight different dimensions that included measures thought to assess anxiety levels (e.g., time in periphery) as well as measures of global locomotor activity (e.g., total numbers of corners or quadrants visited). A One-Way MANOVA revealed no significant group differences (Wilks' $\lambda_{(9,69)}=1.34, p=0.232$ ). These negative results suggest that if behavioral differences are detected between Resilient and PTSD-like rats in the object or objectplace recognition tasks, they are unlikely to reflect disparities in exploratory behaviors or anxiety levels.

Table 1 | Comparison between the behavior of resilient and PTSD-like rats in the open field.

\begin{tabular}{lcc}
\hline & Resilient $(\boldsymbol{n}=\mathbf{3 5})$ & PTSD-like $(\boldsymbol{n}=\mathbf{4 4})$ \\
\hline Time in the center & $37.26 \pm 7.77$ & $41.57 \pm 9.86$ \\
Time in the periphery & $261.57 \pm 7.82$ & $256.57 \pm 9.90$ \\
Latency to leave the center & $31.51 \pm 6.56$ & $39.16 \pm 9.94$ \\
Number of stretch attends & $6.60 \pm 0.65$ & $6.43 \pm 0.5$ \\
Number of rears & $6.31 \pm 0.63$ & $6.34 \pm 0.58$ \\
Number of corners visited & $5.74 \pm 0.86$ & $4.98 \pm 0.76$ \\
Number of quadrants visited & $5.91 \pm 0.81$ & $4.98 \pm 0.73$ \\
Time in the corners & $223 \pm 9.62$ & $233.93 \pm 9.78$ \\
\hline
\end{tabular}

Values are expressed in seconds. Note that two resilient rats had to be excluded due to technical difficulties with the camera.

\section{INCIDENCE OF RESILIENT AND PTSD-LIKE RATS IN THE THREE TASKS}

Distinct samples of Lewis rats were tested in the three object or object-place recognition tasks (NOR, $n=31$; EOR, $n=36$; AOR; $n=48)$. Two days after task completion, they were subjected to predatory threat and tested on the EPM one week later. We aimed to obtain samples that included at least 8-12 PTSD-like rats for each task. Every week, 4-8 rats underwent the paradigm until we reached the target number of PTSD-like rats for each task. However, unexpected differences in the incidence of the PTSDlike phenotype in the three tasks required that different sample sizes be used to reach the target of 8-12 PTSD-like rats in the three tasks.

Indeed, the incidence of the PTSD-like vs. Resilient phenotypes differed markedly between groups (Figure 2A, Chi-square test, $p=0.002$ ). In the NOR sample, $39 \%$ of Lewis rats (or 12 rats of 31) exhibited the PTSD-like phenotype, consistent with the high incidence seen in earlier studies (Cohen et al., 2006a; Goswami et al., 2010) and in the sample used for the OF test. In contrast, the incidence of PTSD-like rats was $25 \%$ in the EOR (or 9 rats of 36) and AOR (or 12 rats of 48) tasks (Figure 2A). Thus, it appears that some aspect of the EOR or AOR tasks, perhaps the increased handling of the rats, reduces the incidence of the PTSD-like phenotype. Consistent with this, when we compared the rat groups subjected to little (OF, NOR) or more extensive handling (EOR, AOR), a significant difference in the relative incidence of the PTSD-like and Resilient phenotypes was observed (Chi-square test, $p=0.0004$ ). This phenomenon is reminiscent of prior studies that described how some stressors or early life experiences can have protective effects on subsequent susceptibility to emotional challenges (Parker and Maestripieri, 2011).

\section{COMMON PATTERNS OF EXPLORATORY BEHAVIORS IN THE NOR, EOR, AND AOR TASKS}

The exploratory behavior of Lewis rats on the various tasks was similar in many respects. We first describe these similarities and then highlight differences between PTSD-like and Resilient rats. Figure 2B shows the time spent by Resilient (blue) and PTSDlike (red) rats exploring the objects during the $5 \mathrm{~min}$ sampling period. A two-factor analysis of variance (ANOVA) using task identity (NOR, EOR, AOR) and behavioral phenotype (PTSDlike, Resilient) as between factors revealed no main effects of task $[F=1.61, d f=2, p=0.21]$ or phenotype $[F=1.64, d f=1$, $p=0.20]$ and no interactions between task and phenotype $[F=$ $2.45, d f=2, p=0.09]$. Consistent with this, we found no significant difference in total exploration of the objects between Resilient and PTSD-like rats during the sample phase of all tasks were combined ( $t$-test, $t=-1.54, d f=112, p=0.13$ ).

In studies using object or object-place recognition tasks, it is customary to compare exploration of the novel and familiar objects (or object-place configurations) over the entire test phase. However, this approach assumes that the pattern of exploration is consistent across the duration of the test phase. We tested this assumption by comparing exploration of the novel (Figure 2C1) and familiar items (Figure 2C2) during the first (F, left) and last ( $\mathrm{L}$, right) minute of the test phases in the three tasks. As shown in Figures 2C1,2, irrespective of group identity, Lewis rats spent a similar amount of time exploring the novel and familiar items 

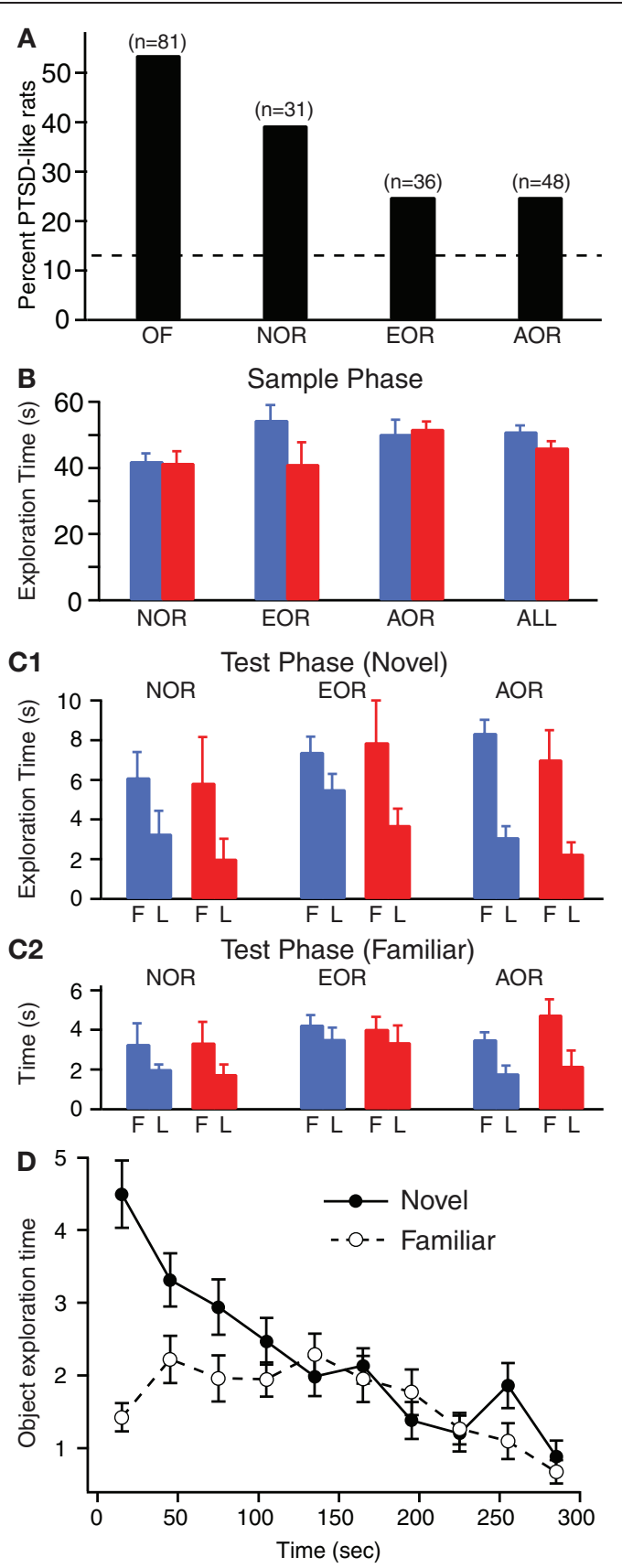

FIGURE 2 | Incidence of PTSD-like phenotype and common patterns of exploratory behavior across the NOR, EOR, and AOR tasks. (A)

Different samples of rats were tested on the EPM one week after predatory threat and classified as PTSD-like if they failed to explore the open arms. Depending on the sample (n's above each bar), prior to predatory threat, the rats were either subjected to the open field test (OF) or one of the object (NOR) or object-place recognition (EOR, AOR) tasks. (B) Total time exploring objects during the sample phase of the three tasks in Resilient (blue) vs. PTSD-like (red) rats. Data obtained in the three rat samples is combined on the right (ALL). (C) Time exploring novel (C1) or familiar (C2) item during the first (F) and last minute (L) of the testing phase in Resilient (blue) vs. PTSD-like (red) rats. (D) Fluctuations in time spent exploring the novel (solid circles and continuous line) or familiar (empty circles and dashed line) item during the test phase of the AOR task. The data is plotted in $30 \mathrm{~s}$ bins during the test phase of the three tasks. Also, there was an overall tendency for the subjects to spend more time exploring the objects early than late in the $5 \mathrm{~min}$ testing period, irrespective of task or phenotype. However, the latter trend appeared to be differentially expressed in relation to the novel (Figure 2C1) vs. familiar (Figure 2C2) items. In particular, the difference between early and late exploration times was markedly higher for the novel than the familiar item. This was confirmed by a 3 (task) by 2 (phenotype) by 2 (object identity) by 2 (exploration period) MANOVA using task identity (NOR, EOR, AOR) and behavioral phenotype (PTSD-like, Resilient), as between factors and object identity (novel, familiar) and exploration period (early, late) as withinsubject factors. This revealed a main effect of object identity $[F=26.6, d f=1, p<0.001]$ and exploration period $[F=46.6$, $d f=1, p<0.001]$ with a significant interaction between them $[F=8.6, d f=1, p=0.004]$, but no effect of phenotype $[F=$ $0.5, d f=1, p=0.51]$. There was also a main effect of task $[F=$ $3.8, d f=2, p=0.025$ ] such that rats showed lower exploration times of the novel and familiar items in the NOR as compared to both EOR and AOR tasks (Scheffe, $p$ 's $<0.015$ ). However, there was no interaction with phenotype (Task-Phenotype interaction $[F=0.05, d f=2, p=0.95])$.

Overall, the above suggests that irrespective of behavioral phenotype, Lewis rats spend more time exploring the items early than late in the test phase and that this effect is more pronounced for the novel items. A better appreciation of this non-uniformity can be gained by examining Figure 2D where we plot fluctuations in the exploration times of the novel (solid line) vs. familiar (dashed line) object-place configurations for all rats subjected to the AOR task. In this and other tasks, it is obvious that preferential exploration of the novel item is maximal during the early part of the test phase and that it decays later. Importantly, exploration of the novel and familiar items becomes nearly indistinguishable toward the end of the test phase. Thus, in order to enhance the sensitivity of comparisons between novel vs. familiar item explorations, it is important to target the early portion of the test phase.

\section{TASK-DEPENDENT DIFFERENCES IN RELATIVE TIME EXPLORING NOVEL vs. FAMILIAR ITEMS}

A frequently used approach to quantify differential object exploration in NOR, EOR, and AOR tasks is to compute a discrimination index (DI). The DI normalizes differences in exploration times between novel and familiar items by the combined exploration time of both items. This minimizes the impact of inter-individual differences in locomotor activity. The DI is computed using the following equation: (Novel - Familiar)/(Novel + Familiar). We adopted this approach to compare the exploratory behavior of Resilient and PTSD-like rats during the test phase of the three tasks. However, note that DIs were not normally distributed in two of the three tasks (Kolmogorov-Smirnov tests, $p<0.05)$. Thus, non-parametric statistical tests are used to assess this data.

Because differential exploration of the novel and familiar items was maximal during the first $1.5 \mathrm{~min}$ of the task (Figure 2D), we used the data obtained during this period to compare the two groups (Figure 3: Resilient, blue; PTSD-like, red). In all three tasks, Lewis rats had positive average DIs indicating preferential 


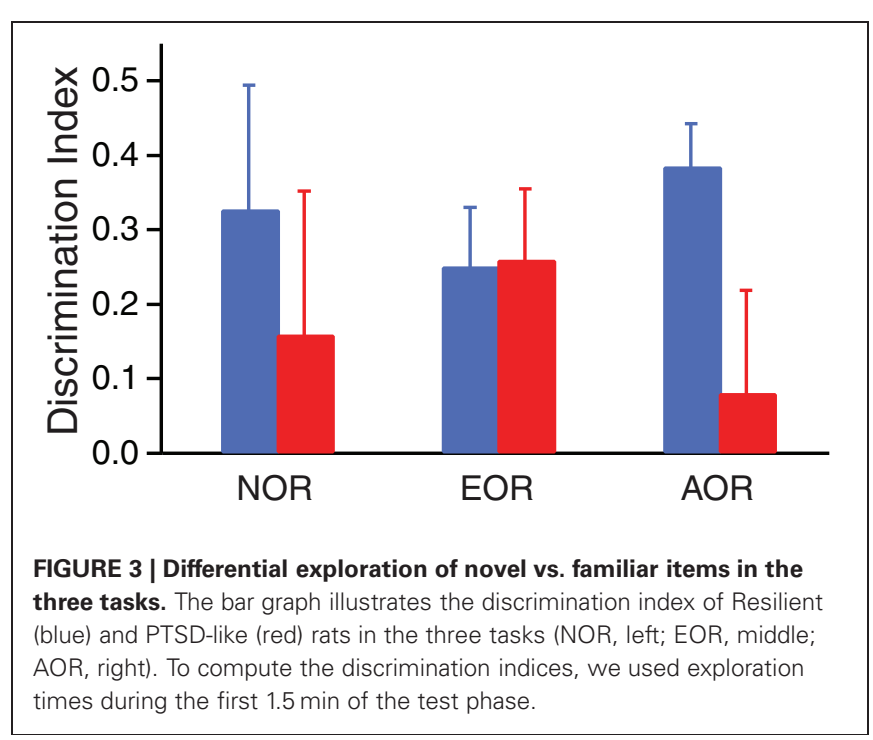

exploration of the novel items irrespective of the type of information required to assess item familiarity/novelty. This was confirmed by comparing the proportion of rats with DIs $>$ or $\leq$ 0 to a random (50:50) distribution (Chi-square, $p<0.001$ ). However, when the proportion of rats with DIs $>$ or $\leq 0$ was compared between the two phenotypes after collapsing the data in the three tasks, a significant difference emerged (Chi-square, $p=0.035$ ). Insights into the origin of this effect can be obtained by comparing the DIs of Resilient and PTSD-like animals in the three tasks. As shown in Figure 3, differences in DIs relative to inter-individual variability seemed negligible in the NOR and EOR tasks, but substantial in the AOR task. Consistent with this, a Mann-Whitney U test comparing the DIs of Resilient vs. PTSD-like rats on the AOR task revealed a significant difference ( $p=0.024)$ with PTSD-like rats exhibiting lower discrimination of the novel items than Resilient rats. Note that the trend apparent in the NOR task was driven by one extreme subject of the PTSDlike group that nearly spent all of the available time during the test phase exploring the familiar object. When this extreme subject was excluded, the trend vanished (DIs: PTSD-like $0.25 \pm 0.17$; Resilient $0.32 \pm 0.16$ ). Consistent with this, when we compared the proportion of PTSD-like vs. resilient rats with DIs $>$ or $\leq 0$ in the NOR task, it did not approach significance (Fisher test, $p=0.28)$.

\section{DISCUSSION}

Ethical limitations inherent to human studies hinder progress in understanding the pathophysiology of PTSD. One approach to circumvent this problem is to study this disorder in a valid animal model of the human syndrome, allowing the use of more invasive techniques than possible in humans. Accordingly, the present study aimed to assess the validity of a rodent model of PTSD, focusing on whether it reproduces the hippocampusassociated deficits seen in the human syndrome. Our results suggest that even prior to trauma, PTSD-like rats show a deficit in hippocampal-dependent functions, as reported in human PTSD. The significance of this observation is considered below.

\section{PRIOR WORK ON THE LEWIS RAT MODEL OF PTSD}

A promising line of animal research has focused on the impact of species-relevant threatening stimuli that mimic the type of lifeand-death experiences known to precipitate PTSD in humans. It was observed that rodents exposed to predators or their odor develop enduring ( $\geq 3$ weeks) signs of anxiety as determined with several behavioral assays such as the EPM, acoustic startle, and social interaction test (Adamec and Shallow, 1993; Blanchard et al., 2003; Adamec et al., 2006). As in human PTSD, differential susceptibility to predatory threat was seen in rodents. For instance, Cohen et al. (2006b) reported that following predatory threat, the incidence of extreme behavioral manifestations of anxiety varied markedly in different rat strains: $50 \%$ in Lewis rats compared to $20 \%$ of Sprague-Dawley and $10 \%$ of Fisher rats. The equal prevalence of susceptible and resilient subjects among Lewis rats led us to focus on this strain. We reasoned that because random groups of Lewis rats include a roughly equal proportion of susceptible and resilient subjects, fewer rats would have to be studied to compare the two groups on any dimension. This would be particularly advantageous for labor-intensive studies such as those relying on single-unit recordings in behaving animals.

Thus, in an earlier study (Goswami et al., 2010), we began assessing the validity of the Lewis rat model of PTSD. We first tested whether this model replicates the compromised ability of human PTSD subjects to extinguish conditioned fear responses, a trait thought to play a critical role in the persistence of PTSD (reviewed in Quirk and Mueller, 2008). Indeed, humans with PTSD exhibit an extinction deficit (Orr et al., 2000; Peri et al., 2000; Blechert et al., 2007; Milad et al., 2008, 2009). Importantly, a study of identical twins discordant for trauma exposure revealed that this deficit develops as a result of trauma and does not predate it (Milad et al., 2008). Consistent with the human data, we observed that PTSD-like rats showed a clear extinction deficit if predatory threat occurred before, but not after, fear conditioning (Goswami et al., 2010). In contrast, the relative timing of predatory threat and fear conditioning did not change the properties of extinction in resilient rats (Goswami et al., 2010). This pattern of results suggested that the extinction deficit manifested by PTSDlike rats is not an antecedent condition but is acquired as a result of predatory threat, paralleling human findings.

\section{DIFFERENTIAL PERFORMANCE OF RESILIENT AND SUSCEPTIBLE RATS IN OBJECT RECOGNITION TASKS}

A vast literature indicates that individuals with PTSD are impaired on hippocampal-dependent tasks (for instance, see Shin et al., 2004; Lindauer et al., 2006; Gilbertson et al., 2007; Thomaes et al., 2009; Hayes et al., 2011; reviewed in Samuelson, 2011). Moreover, studies of monozygotic twins discordant for combat exposure have revealed that hippocampal abnormalities predate onset of the disorder (Gilbertson et al., 2002, 2007), in contrast with the fear extinction deficit (Milad et al., 2008). Therefore, the present study tested whether the Lewis rat model of PTSD reproduces the hippocampal deficit seen in human PTSD, and whether it can be detected before exposure to predatory threat.

To assess hippocampal functioning, we used three different recognition tasks (NOR, EOR, AOR) where subjects manifest that they have previously encountered objects or object-place 
configurations by preferential exploration of novel items. It should be mentioned that there is significant debate in the literature regarding the hippocampal dependence of the three tasks (Mumby, 2001; Langston and Wood, 2009). However, we note that recognition of item familiarity using an allocentric frame of reference, as in our AOR task, seems to be particularly dependent on hippocampal functioning in rats (Langston and Wood, 2009) and humans (Suthana et al., 2009). Importantly, the exploratory behavior of resilient and PTSD-like rats was indistinguishable in a novel OF and during the sampling phase of the three tasks. This suggests that there were no disparities between two rat groups in their initial object investigations. In contrast, during the test phase, the performance of PTSD-like rats was inferior to that of resilient rats, but only on the AOR task. That is, PTSD-like rats spent less time exploring the novel relative to the familiar object-place configuration than resilient rats. In light of prior work indicating that hippocampal functioning is especially critical for detecting familiarity in an allocentric spatial frame of reference (Langston and Wood, 2009; Suthana et al., 2009), these results support the notion that PTSD-like rats reproduce the hippocampal deficit seen in human PTSD.

Further support for the hippocampal-dependent nature of the AOR task can be found in the literature that explores the effects of stress on striatal- and hippocampal-dependent learning strategies (e.g., Packard and Wingard, 2004; Schwabe et al., 2007; Schwabe and Wolf, 2009). There is converging evidence that animals and humans show preference for striatal-dependent (or response learning), as opposed to hippocampal-dependent (or place learning) under conditions of stress. The mechanisms for this switch remain unclear but it is thought to be related to the

\section{REFERENCES}

Adamec, R., Head, D., Blundell, J., Burton, P., and Berton, O. (2006). Lasting anxiogenic effects of feline predator stress in mice: sex differences in vulnerability to stress and predicting severity of anxiogenic response from the stress experience. Physiol. Behav. 88, 12-29.

Adamec, R. E., and Shallow, T. (1993). Lasting effects on rodent anxiety of a single exposure to a cat. Physiol. Behav. 54, 101-109.

Afifi, T. O., Asmundson, G. J., Taylor, S., and Jang, K. L. (2010). The role of genes and environment on trauma exposure and posttraumatic stress disorder symptoms: a review of twin studies. Clin. Psychol. Rev. 30, 101-112.

Blanchard, D. C., Griebel, G., and Blanchard, R. J. (2003). Conditioning and residual emotionality effects of predator stimuli: some reflections on stress and emotion. Prog. Neuropsychopharmacol. Biol. Psychiatry 27, 1177-1185.

Blanchard, R. J., Yang, M., Li, C. I., Gervacio, A., and Blanchard, D.
C. (2001). Cue and context conditioning of defensive behaviors to cat odor stimuli. Neurosci. Biobehav. Rev. 25, 587-595.

Blechert, J., Michael, T., Vriends, N., Margraf, J., and Wilhelm, F. H. (2007). Fear conditioning in posttraumatic stress disorder: evidence for delayed extinction of autonomic, experiential, and behavioural responses. Behav. Res. Ther. 45, 2019-2033.

Bremner, J. D., Vythilingam, M., Vermetten, E., Southwick, S. M., McGlashan, T., Nazeer, A., Khan, S., Vaccarino, L. V., Soufer, R., Garg, P. K., Ng, C. K., Staib, L. H., Duncan, J. S., and Charney, D. S. (2003). MRI and PET study of deficits in hippocampal structure and function in women with childhood sexual abuse and posttraumatic stress disorder. Am. J. Psychiatry 160, 924-932.

Cohen, H., Matar, M. A., RichterLevin, G., and Zohar, J. (2006a). The contribution of an animal model toward uncovering biological risk factors for PTSD. Ann. N.Y. Acad. Sci. 1071, 335-350. release of stress hormones (Schwabe et al., 2009). While task completion in the present study preceded stress, these data support the idea that rats predisposed toward PTSD may already evidence a hippocampal deficit, which creates a preference toward striataldependent learning. Future studies should relate these behavioral findings to hippocampal volumes or other deficits to validate these findings.

While future studies should examine hippocampal and fear extinction deficits within the same rat sample, it is important to consider the impact of handling on the incidence of the PTSDlike phenotype. Indeed, because nearly half the PTSD-like rats are somehow protected against the long-term impact of predatory threat exposure when they are subjected to extensive handling prior to the stressor, such rats would be classified as Resilient, yet they might still exhibit hippocampal or extinction deficits. However, this remains to be determined. The same difficulty likely contributed to modest differences found between PTSD-like and resilient rats in the present study. These are critical issues that should be addressed in subsequent studies.

Nevertheless, combined with our prior findings on fear extinction (Goswami et al., 2010), the present results suggest that the Lewis rat model of PTSD has face validity. Therefore, comparing limbic neuronal interactions in resilient vs. at risk Lewis rats might shed light on the causes and pathophysiology of human PTSD.

\section{ACKNOWLEDGMENTS}

We thank Davine Armstrong for help with scoring of the open field behavior. This material is based upon work supported by NIMH grant RO1 MH083710 to Denis Paré.
Cohen, H., Zohar, J., Gidron, Y., Matar, M. A., Belkind, D., Loewenthal, U., Kozlovsky, N., and Kaplan, Z. (2006b). Blunted HPA axis response to stress influences susceptibility to posttraumatic stress response in rats. Biol. Psychiatry 59, 1208-1218. Gilbertson, M. W., Shenton, M. E. Ciszewski, A., Kasai, K., Lasko, N. B., Orr, S. P., and Pitman, R. K. (2002). Smaller hippocampal volume predicts pathologic vulnerability to psychological trauma. Nat. Neurosci. 5, 1242-1247.

Gilbertson, M. W., Williston, S. K., Paulus, L. A., Lasko, N. B., Gurvits, T. V., Shenton, M. E., Pitman, R. K., and Orr, S. P. (2007). Configural cue performance in identical twins discordant for posttraumatic stress disorder: theoretical implications for the role of hippocampal function. Biol. Psychiatry 62, 513-520.

Goswami, S., Cascardi, M., RodriguezSierra, O. E., Duvarci, S., and Pare, D. (2010). Impact of predatory threat on fear extinction in lewis rats. Learn. Mem. 17, 494-501.

Hayes, J. P., LaBar, K. S., McCarthy, G., Selgrade, E., Nasser, J., Dolcos, F., and Morey, R. A. (2011). Reduced hippocampal and amygdala activity predicts memory distortions for trauma reminders in combatrelated PTSD. J. Psychiatr. Res. 45, 660-669.

Kitayama, N., Vaccarino, V., Kutner, M., Weiss, P., and Bremner, J. D. (2005). Magnetic resonance imaging (MRI) measurement of hippocampal volume in posttraumatic stress disorder: a meta-analysis. J. Affect. Disord. 88, 79-86.

Langston, R. F., and Wood, E. R. (2009). Associative recognition and the hippocampus: differential effects of hippocampal lesions on object-place, object-context, and object-place-context memory. Hippocampus 20, 1139-1153.

Lindauer, R. J., Olff, M., van Meijel, E. P., Carlier, I. V., and Gersons, B. P. (2006). Cortisol, learning, memory, and attention in relation to smaller hippocampal volume in police officers with posttraumatic stress disorder. Biol. Psychiatry 59, 171-177.

McGregor, I. S., Schrama, L., Ambermoon, P., and Dielenberg, R. A. (2002). Not all 'predator odours' 
are equal: cat odour but not 2, 4, 5 trimethylthiazoline (TMT; fox odour) elicits specific defensive behaviours in rats. Behav. Brain Res. 129, 1-16.

Milad, M. R., Orr, S. P., Lasko, N. B., Chang, Y., Rauch, S. L., and Pitman, R. K. (2008). Presence and acquired origin of reduced recall for fear extinction in PTSD: results of a twin study. J. Psychiatr. Res. 42, 515-520.

Milad, M. R., Pitman, R. K., Ellis, C. B., Gold, A. L., Shin, L. M., Lasko, N. B., Zeidan, M. A., Handwerger, K., Orr, S. P., and Rauch, S. L. (2009). Neurobiological basis of failure to recall extinction memory in posttraumatic stress disorder. Biol. Psychiatry 66, 1075-1082.

Mumby, D. G. (2001). Perspectives on object-recognition memory following hippocampal damage: lessons from studies in rats. Behav. Brain Res. 127, 159-181.

Nemeroff, C. B., Bremner, J. D., Foa, E. B., Mayberg, H. S., North, C. S., and Stein, M. B. (2006). Posttraumatic stress disorder: a state-of-thescience review. J. Psychiatr. Res. 40, $1-21$.

Nugent, N. R., Amstadter, A. B., and Koenen, K. C. (2008). Genetics of post-traumatic stress disorder: informing clinical conceptualizations and promoting future research. Am. J. Med. Genet. C Semin. Med. Genet. 148C, 127-132.
Orr, S. P., Metzger, L. J., Lasko, N. B., Macklin, M. L., Peri, T., and Pitman, R. K. (2000). De novo conditioning in trauma-exposed individuals with and without posttraumatic stress disorder. J. Abnorm. Psychol. 109, 290-298.

Packard, M. G., and Wingard, J. C. (2004). Amygdala and "emotional" modulation of the relative use of multiple memory systems. Neurobiol. Learn. Mem. 82, 243-252.

Parker, K. J., and Maestripieri, D. (2011). Identifying key features of early stressful experiences that produce stress vulnerability and resilience in primates. Neurosci. Biobehav. Rev. 35, 1466-1483.

Peri, T., Ben-Shakhar, G., Orr, S. P., and Shalev, A. Y. (2000). Psychophysiologic assessment of aversive conditioning in posttraumatic stress disorder. Biol. Psychiatry 47, 512-519.

Quirk, G. J., and Mueller, D. (2008). Neural mechanisms of extinction learning and retrieval. Neuropsychopharmacology 33, 56-72.

Samuelson, K. W. (2011). Posttraumatic stress disorder and declarative memory functioning: a review. Dialogues Clin. Neurosci. 13, 346-351.

Schwabe, L., Oitzl, M. S., Philippsen, C., Richter, S., Bohringer, A., Wippich, W., and Schachinger, H. (2007).
Stress modulates the use of spatial and stimulus response learning strategies in humans. Learn. Mem. 14, 109-116.

Schwabe, L., Schachinger, H., De Kloet, E. R., and Oitzl, M. S. (2009). Corticosteroids operate as switch between memory systems. J. Cogn. Neurosci. 22, 1362-1372.

Schwabe, L., and Wolf, O. T. (2009). Stress prompts habit behavior in humans. J. Neurosci. 29, 7191-7198.

Shin, L. M., Shin, P. S., Heckers, S., Krangel, T. S., Macklin, M. L., Orr, S. P., Lasko, N., Segal, E., Makris, N., Richert, K., Levering, J., Schacter, D. L., Alpert, N. M., Fischman, A. J., Pitman, R. K., and Rauch, S. L. (2004). Hippocampal function in posttraumatic stress disorder. Hippocampus 14 292-300.

Suthana, N. A., Ekstrom, A. D., Moshirvaziri, S., Knowlton, B., and Bookheimer, S. Y. (2009). Human hippocampal CA1 involvement during allocentric encoding of spatial information. J. Neurosci. 29, 10512-10519.

Thomaes, K., Dorrepaal, E., Draijer, N. P., de Ruiter, M. B., Elzinga, B. M., van Balkom, A. J., Smoor, P. L., Smit, J., and Veltman, D. J. (2009). Increased activation of the left hippocampus region in complex PTSD during encoding and recognition of emotional words: a pilot study. Psychiatry Res. 171, 44-53.

Wang, Z., Neylan, T. C., Mueller, S. G., Lenoci, M., Truran, D., Marmar, C. R., Weiner, M. W., and Schuff, N. (2010). Magnetic resonance imaging of hippocampal subfields in posttraumatic stress disorder. Arch. Gen. Psychiatry 67, 296-303.

Conflict of Interest Statement: The authors declare that the research was conducted in the absence of any commercial or financial relationships that could be construed as a potential conflict of interest.

Received: 19 April 2012; paper pending published: 15 May 2012; accepted: 24 May 2012; published online: 12 June 2012.

Citation: Goswami S, Samuel S, Sierra OR, Cascardi $M$ and Paré D (2012) A rat model of post-traumatic stress disorder reproduces the hippocampal deficits seen in the human syndrome. Front. Behav. Neurosci. 6:26. doi: 10.3389/ fnbeh.2012.00026

Copyright (c) 2012 Goswami, Samuel, Sierra, Cascardi and Paré. This is an open-access article distributed under the terms of the Creative Commons Attribution Non Commercial License, which permits non-commercial use, distribution, and reproduction in other forums, provided the original authors and source are credited. 